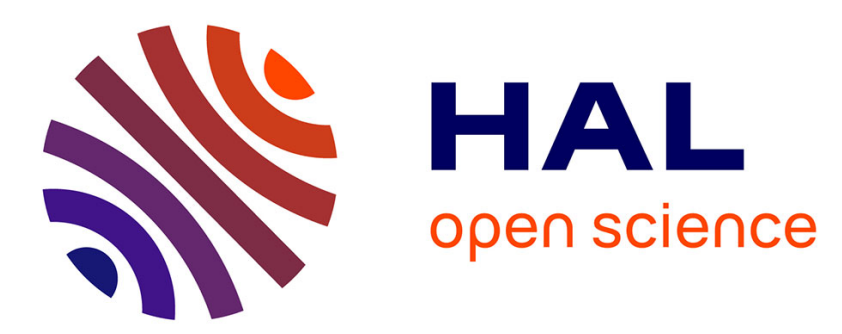

\title{
Propriétés magnétiques de ferrites CoMnxFe2-xO4 (0x1) à structure spinelle
}

\author{
I. Chassaing, L. Presmanes, Ph. Tailhades, A. Rousset, P. Mollard
}

\section{To cite this version:}

I. Chassaing, L. Presmanes, Ph. Tailhades, A. Rousset, P. Mollard. Propriétés magnétiques de ferrites CoMnxFe2-xO4 (0x1) à structure spinelle. Journal de Physique IV Proceedings, 1992, 02 (C3), pp.C3127-C3-131. 10.1051/jp4:1992319 . jpa-00251524

\section{HAL Id: jpa-00251524 https://hal.science/jpa-00251524}

Submitted on 1 Jan 1992

HAL is a multi-disciplinary open access archive for the deposit and dissemination of scientific research documents, whether they are published or not. The documents may come from teaching and research institutions in France or abroad, or from public or private research centers.
L'archive ouverte pluridisciplinaire HAL, est destinée au dépôt et à la diffusion de documents scientifiques de niveau recherche, publiés ou non, émanant des établissements d'enseignement et de recherche français ou étrangers, des laboratoires publics ou privés. 


\title{
Propriétés magnétiques de ferrites $\mathrm{CoMn}_{\mathbf{x}} \mathrm{Fe}_{2-\mathrm{x}} \mathrm{O}_{4}(0 \leq x \leq 1)$ à structure spinelle
}

\author{
I. CHASSAING, L. PRESMANES, Ph. TAILHADES, A. ROUSSET et P. MOLLARD
}

Laboratoire de Chimie des Materiaux Inorganiques, URA-CNRS 1311, Université P. Sabatier, 118 Rte de Narbonne, 31062 Toulouse cedex, France

"Laboratoire L. Néel, CNRS, Avenue des Martyrs, 166X, 38042 Grenoble cedex, France

\begin{abstract}
The magnetic properties of Co-Mn spinel ferrites depend on Mn content, oxidation degree, cationic distribution and morphology. Slow cooling after oxidation treatment at $350^{\circ} \mathrm{C}$ allows to increase the coercivity by the creation of a directional order and migration of the $\mathrm{Co}^{2+}$ ions from A sites to B sites.
\end{abstract}

\section{INTRODUCTION}

Les magnétites substituées par du cobalt et du manganèse présentent des propriétés intéressantes pour l'enregistrement magnétique et magnétooptique. Leurs caractéristiques magnétiques, notamment le champ coercitif, sont fortement dépendantes de leur composition, du degré d'oxydation des cations et de la distribution cationique. Les études effectuées sur les particules d'oxyde $\mathrm{CoMn}_{\mathrm{x}} \mathrm{Fe}_{2-\mathrm{x}} \mathrm{O}_{4+\mathrm{d}}(0 \leq x \leq 1 ; 0 \leq \mathrm{d} \leq 0,5)$ ont permis d'établir les différents états de valence du manganèse et les formules structurales de ces composés [1]. Les phases stoechiométriques $(d=0)$ sont décrites par la formule structurale suivante:

$$
\mathrm{Co}_{\mathrm{a}}^{2+} \mathrm{Fe}_{1-\mathrm{a}}^{3+}\left[\mathrm{Fe}_{1-\mathrm{x}+\mathrm{a}}^{3+} \mathrm{Co}_{1-\mathrm{a}}^{2+} \mathrm{Mn}_{\mathrm{x}}^{3+}\right] \mathrm{O}_{4}^{2-}
$$

(a est la fraction d'ions $\mathrm{Co}^{2+}$ en site tétraédrique)

Les ions $\mathrm{Mn}^{3+}$ peuvent être partiellement oxydés entre $250^{\circ} \mathrm{C}$ et $400^{\circ} \mathrm{C}$ au sein même de la structure spinelle selon $4 \mathrm{Mn}^{3+}-\mathrm{Mnn}^{4+}+\square$ La distribution cationique de ces phases lacunaires est alors:

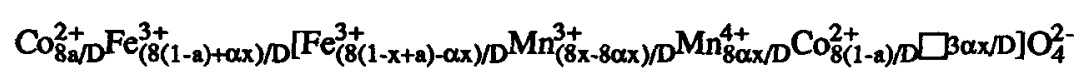

$\left(\mathrm{D}=8+\alpha x ; \alpha\right.$ est la fraction d'ions $\mathrm{Mn}^{3+}$ oxydés)

Par ailleurs, l'occupation préferentielle des sites octaédriques par les ions $\mathrm{Mn}^{3+}$ et $\mathrm{Mn}^{4+}$ entraîne une migration partielle des ions $\mathrm{Co}^{2+}$ des sites octaédriques vers les sites tétraédriques [1], en accord avec les travaux de Blasse pour des ferrites préparés à hautes températures [2].

Les travaux de Néel [3] ont mis en évidence l'existence de réarrangements cationiques locaux attribués à l'établissement d'un ordre directionnel. La création des structures anisotropes est fonction de la composition et de la cinétique de mise en équilibre, laquelle varie considérablement avec la température. Les performances magnétiques des oxydes peuvent être fortement améliorées à l'aide de traitements thermiques appropriés. En effet, 
des gains de coercivité importants ont été observés pour des ferrites $\mathrm{Co}_{y} \mathrm{Mn}_{x} \mathrm{Fe}_{3-x-y} \mathrm{O}_{4}(0,2<x<1 ; y=0,08 ; 0,18)$ oxydés à $350^{\circ} \mathrm{C}$ et refroidis lentement ou recuits à des températures proches de $270^{\circ} \mathrm{C}$ [4]. Ces accroissements ont été attribués à la création d'un ordre directionnel [3], [5], [6].

Dans ce travail, nous étudions l'évolution des propriétés magnétiques des ferrites $\mathrm{CoMn}_{\mathrm{x}} \mathrm{Fe}_{2-\mathrm{x}} \mathrm{O}_{4+\mathrm{d}}(0 \leq x \leq 1)$ en fonction des traitements thermiques (température, vitesse de refroidissement) et de la composition en manganèse $x$.

PREPARATION DES ECHANTILLONS ET TECHNIQUES EXPERIMENTALES.

Les particules aciculaires $\mathrm{CoMn}_{\mathbf{x}} \mathrm{Fe}_{2-\mathrm{x}} \mathrm{O}_{4+d}$ sont synthétisées à partir de précurseurs oxaliques $\mathrm{Co}_{1 / 3} \mathrm{Mn}_{x / 3} \mathrm{Fe}(2-x) / 3 \mathrm{C}_{2} \mathrm{O}_{4}, 2 \mathrm{H}_{2} \mathrm{O}$ précipités en milieu alcoolique. Les phases pures a structure spinelle sont obtenues directement aprés une décomposition lente des oxalates sous air jusqu'à $600^{\circ} \mathrm{C}$. Les compositions sont présentées dans le tableau 1.

tableau 1: Teneurs en cobalt et en manganèse des ferrites $\mathrm{CoMn}_{\mathrm{x}} \mathrm{Fe}_{2-\mathrm{x}} \mathrm{O}_{4}$

\begin{tabular}{|c|c|c|c|}
\hline & \%Mn (masse) & \%Co (masse) & Formule brute \\
\hline $\bar{A}$ & 0 & 25,1 & $\mathrm{Co}_{1} \mathrm{Fe}_{2} \mathrm{O}_{4}$ \\
\hline$\overline{\mathbf{K}}$ & 2,8 & 25,1 & $\mathrm{ColMn}_{0,12} \mathrm{Fe}_{1,88} \mathrm{O}_{4}$ \\
\hline$\overline{\mathbf{P}}$ & 9,42 & 23,5 & $\mathrm{Co}_{0,93} \mathrm{Mn}_{0,4} \mathrm{Fe}_{1,67} \mathrm{O}_{4}$ \\
\hline $\bar{S}$ & 10,6 & 24,6 & 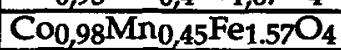 \\
\hline $\mathrm{T}$ & 15,24 & 25,06 & $\mathrm{Co}_{1} \mathrm{Mn}_{0,65} \mathrm{Fe}_{1,35} \mathrm{O}_{4}$ \\
\hline$\overline{\mathbf{Z}}$ & 18,6 & 25,94 & $\mathrm{Co}_{1,03} \mathrm{Mn}_{0,79 \mathrm{Fe}_{1,18} \mathrm{O}_{4}}$ \\
\hline$\overline{\bar{x}}$ & 22,1 & 26,5 & $\mathrm{Co}_{1,05} \mathrm{Mn}_{0,94} \mathrm{Fe}_{1,01} \mathrm{O}_{4}$ \\
\hline
\end{tabular}

Les particules sont ensuite soumises à:

- des traitements de recuit à des températures proches de $700^{\circ} \mathrm{C}$ afin d'ajuster la taille des cristallites à $45 \mathrm{~nm}$

- des traitements de réduction à $300^{\circ} \mathrm{C}$ sous un mélange d'azote (80\%), d'hydrogène $(8 \%)$ et de vapeur d'eau (12\%) afin d'élaborer des ferrites stoechiométriques.

- des traitements d'oxydation à l'air conduisant à la formation de phases lacunaires.

Les particules submicroniques présentent la même morphologie aciculaire (longueur/diametre $=4$ à 5 ) et la même taille (longueur moyenne $0,2 \mu \mathrm{m}$ )

Les analyses thermogravimétriques sont effectuées sur. un thermoanalyseur Setaram TAG 24 équipé d'une balance symétrique. Les propriétés magnétiques des particules sont déterminées à température ambiante à l'aide d'un hystéresisgraphe M2000 de S2IS permettant d'appliquer un champ magnétique maximum de $25 \mathrm{kOe}$.

\section{RESULTATS ET DISCUSSION}

L'évolution du champ coercitif avec la température d'oxydation a été étudiée dans le cas de magnétites substituées $\mathrm{CoMn}_{x} \mathrm{Fe}_{2-\mathrm{x}} \mathrm{O}_{4}$ oxydées à l'air et trempées (vitesse de refroidissement $=3000^{\circ} \mathrm{C} / \mathrm{h}$ ) (fig.1). La variation de ce paramètre est alors semblable à celle déjà observée pour des ferrites à plus faible teneur en cobalt $\mathrm{Co}_{y} \mathrm{Mn}_{x} \mathrm{Fe}_{3-x-y} \mathrm{O}_{4}$ [4]. Ainsi, l'analogie avec les études 
antérieures [4] et les analyses thermogravimétriques (fig.2) montrent que l'accroissement du champ coercitif est corrélé à l'oxydation des ions $\mathrm{Mn}^{3+}$ en ions $\mathrm{Mn}^{4+}$. La forte décroissance de celui-ci pour des températures supérieures à $350^{\circ} \mathrm{C}$ résulte de la réduction des ions $\mathrm{Mn}^{4+}$. Le décalage en température de ces effets (fig.1 et 2) provient des cinétiques différentes des conditions de traitement (montée en température a $3^{\circ} \mathrm{C} / \mathrm{min}$ en thermogravimétrie et à $2,5^{\circ} \mathrm{C} / \mathrm{min}$ suivie $\mathrm{d}^{\prime}$ un maintien en température de $2 \mathrm{~h}$ lors des traitements d'oxydation).

Le maximum de coercivité observé pour les phases oxydées à des températures proches de $350^{\circ} \mathrm{C}$ est attribué à l'établissement d'un ordre directionnel. En effet, bien que la cinétique de réorganisation soit lente, la teneur importante en lacunes dans les sites octaédriques favorise la migration des ions et peut ainsi contribuer à la création d'arrangements cationiques locaux anisotropes. Lorsque ces derniers impliquent des ions $\mathrm{Co}^{2+}$ a trés fort couplage spin orbite placés en site octaédrique, ils engendrent un accroissement important du champ coercitif [7].

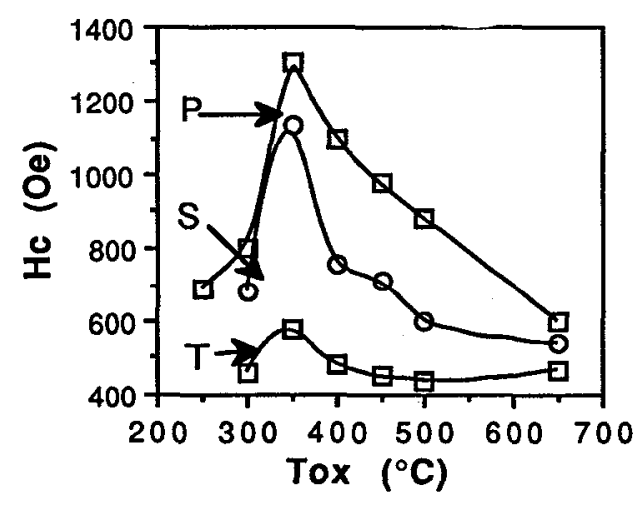

Figure 1: Evoution du champ coercitif avec la température d'oxydation de ferrites $\mathrm{CoMn}_{x} \mathrm{Fe}_{2-x} \mathrm{O}_{4}(0 \leq \mathrm{x} \leq 1)$

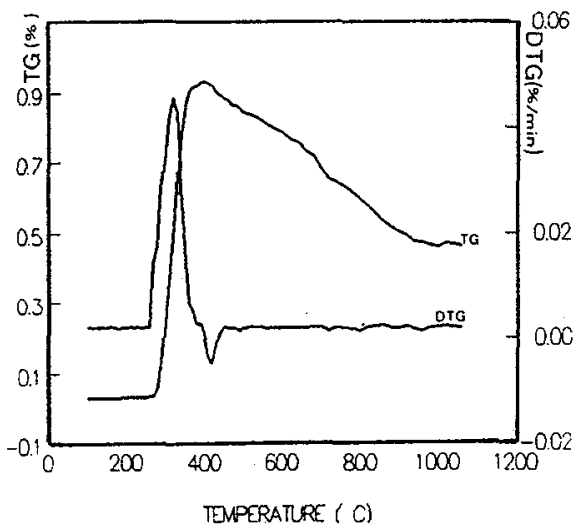

Figure 2: Thermogravimétrie et thermo-gravimétrie différentielle de ferrites $\mathrm{Co}_{0,94} \mathrm{Mn}_{1,05} \mathrm{Fe}_{1,01} \mathrm{O}_{4}$

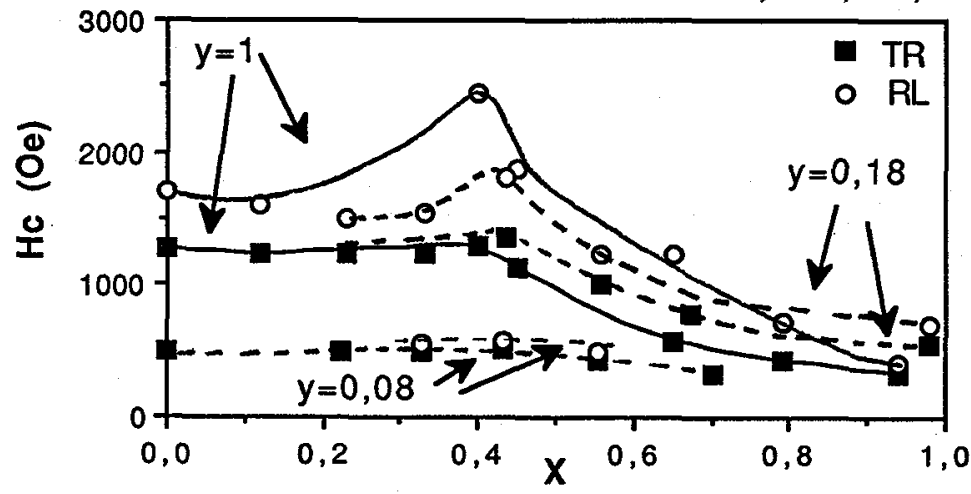

Figure 3: Evolution du champ coercitif $\mathrm{Hc}$ avec la teneur en $\mathrm{Mn}(\mathrm{x})$ pour des phases $\mathrm{CoMn}_{\mathbf{x}} \mathrm{Fe}_{2-\mathrm{x}} \mathrm{O}_{4}$ trempées (TR) et refroidies lentement $(\mathrm{RL})\left(\right.$. Analogie avec les phases $\mathrm{Coy}_{y} \mathrm{Mn}_{x} \mathrm{Fe}_{3-\mathrm{x}-\mathrm{y}} \mathrm{O}_{4}$ $(-)[4]$. 
Compte tenu des fortes coercivités présentées par les phases oxydées à $350^{\circ} \mathrm{C} \mathrm{CoMn} \times \mathrm{Fe}_{2-x} \mathrm{O}_{4}$, cette propriété est étudiée pour des échantillons trempés (TR) et refroidis lentement (RL) renfermant différentes teneurs en manganèse $x$ (fig.3). Une coercivité maximale est atteinte pour des compositions proches de $x=0,45$, indépendamment du traitement thermique. Or, pour cette composition, les particules sont mieux individualisées et ont des textures ainsi que des formes plus régulières et homogènes, que pour les autres échantillons (fig.4). Cette qualité morphologique pourrait être à l'origine du maximum observé. Cependant un champ coercitif optimal a également été observé pour des couches minces de ferrites $\mathrm{CoMn}_{\mathrm{x}} \mathrm{Fe}_{2-\mathrm{x}} \mathrm{O}_{4}$ de teneur $\mathrm{x=0,4}$ dont la morphologie ne présentait aucune spécificité. La morphologie des particules ne semblent donc pas être la seule origine du maximum de coercivité observé (fig.3).

a)

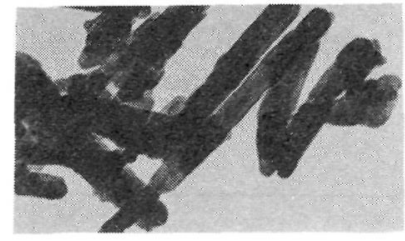

b)

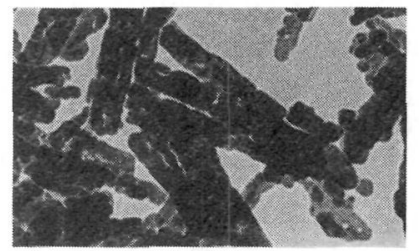

Figure 4: Micrographies de particules aciculaires $\mathrm{CoMn}_{x} \mathrm{Fe}_{2-x} \mathrm{O}_{4}$ observées en microscopie électronique à transmission. (a): $x=0,45 ;(b): x>0,45$.

Par ailleurs, des refroidissements lents $\left(5^{\circ} \mathrm{C} / \mathrm{h}\right)$ effectués aprés les traitements d'oxydation s'accompagnent de gains de coercivité comme pour les ferrites $\mathrm{Co}_{y} \mathrm{Mn}_{x} \mathrm{Fe}_{3-x-y} \mathrm{O}_{4}(y<0,2)$ précedemment étudiés (fig.3). Pour ces derniers, l'exaltation du champ coercitif a été attribuée a la création d'un ordre directionnel [3] [5], aucune modification significative des caractéristiques morphologiques, du paramètre cristallin, ainsi que des aimantations n'ayant été décelées en fonction du mode de refroidissement. Compte tenu des similitudes de comportement et de composition entre les phases $\mathrm{Co}_{\mathbf{y}} \mathrm{Mn}_{\mathbf{x}} \mathrm{Fe}_{3-\mathrm{x}}$ $\mathrm{y}_{4}(\mathrm{y}<0,2)$ et les phases $\mathrm{CoMn}_{\mathrm{x}} \mathrm{Fe}_{2-\mathrm{x}} \mathrm{O}_{4}$, objets de la présente étude, un ordre directionnel doit être également créé dans ces dernières.

Toutefois, les ferrites $\mathrm{CoMn}_{x} \mathrm{Fe}_{2-x} \mathrm{O}_{4}$ présentent, pour une composition donnée, des aimantations à 20kOe différentes, selon qu'ils sont trempés ou refroidis lentement (tabl.2). Cette anomalie, significative en valeur relative de l'évolution de l'aimantation à saturation avec la teneur en manganèse, est absente dans les phases $\mathrm{Co}_{y} \mathrm{Mn}_{\mathrm{x}} \mathrm{Fe}_{3-\mathrm{x}-\mathrm{y}} \mathrm{O}_{4}(\mathrm{y}<0,2)$. L'interprétation suivante est proposée. Ce phénomène peut être attribué à une migration d'ions $\mathrm{Co}^{2+}$ des sites tétraédriques vers les sites octaédriques. En effet lors de l'élaboration, aprés la décomposition des oxalates, les produits sont portés à des températures supérieures a $600^{\circ} \mathrm{C}$ puis refroidis rapidement. A de telles températures il a été établi que des ions $\mathrm{Co}^{2+}$ pouvaient occuper non seulement les sites $\mathrm{B}$, dans lesquels leur energie de stabilisation est la plus grande, mais aussi les sites A [8] [9] [10] [11]. La coordinence 4 d'une partie des ions $\mathrm{Co}^{2+}$ est par ailleurs favorisée par la présence des ions $\mathrm{Mn}^{3+}$ comme l'a signalé Blasse [2]. Les traitements ultérieurs de réduction et d'oxydation suivis d'une trempe sont effectués à des températures modérées $\left(<400^{\circ} \mathrm{C}\right)$ pendant des durées relativement courtes $(<3 \mathrm{~h})$, ils ne permettent donc pas d'atteindre l'état d'équilibre. En revanche, les refroidissements lents aprés oxydation maintiennent pendant plusieurs heures les échantillons à des températures proches de $350^{\circ} \mathrm{C}$. Ils permettent donc un retour progressif des ions $\mathrm{Co}^{2+}$ 
tétraédriques vers les sites octaédriques. Les ferrites de cobalt-manganèse riches en cobalt se distinguent ainsi des matériaux précédemment étudiés [4] [7] par la présence d'un passage des ions $\mathrm{Co2}+$ des sites tétraédriques vers les sites octaédriques, en plus de réarrangements locaux sur les sites octaédriques.

\begin{tabular}{|c|c|c|}
\hline & $M($ uem/g), TR & M (uem/g), RL \\
\hline A & 72 & 70,6 \\
\hline K & 67,2 & 62,9 \\
\hline$\overline{\mathbf{P}}$ & 76 & 56,4 \\
\hline $\mathrm{T}$ & 58,5 & 46,3 \\
\hline $\mathrm{Z}$ & 58,5 & 46,5 \\
\hline $\mathrm{X}$ & 40,6 & 34,2 \\
\hline
\end{tabular}

Tableau 2: Aimantations à 20 kOe mesurées à température ambiante pour les phases oxydées trempées (TR) et refroidies lentement (RL).

\section{CONCLUSION}

L'évolution du champ coercitif des ferrites $\mathrm{CoMn}_{\mathrm{x}} \mathrm{Fe}_{2-\mathrm{x}} \mathrm{O}_{4}$ est fortement dépendante de la teneur en manganèse. La substitution du fer par cet élément offre en effet la possibilité de modifier l'état d'oxydation du ferrite, faisant apparaître des lacunes cationiques. Celles-ci favorisent la rapidité de réorganisation du réseau cristallin et l'établissement d'un ordre directionnel. $\mathrm{Ce}$ dernier semble contribuer, comme pour les phases $\mathrm{Co}_{y} \mathrm{Mn}_{\mathrm{x}} \mathrm{Fe}_{3-x-y} \mathrm{O}_{4}$, à l'obtention de fortes coercivités (2500 Oe). La complexité du comportement magnétique de ces matériaux à valence mixte résulte du nombre de cations présents, des migrations cationiques et des configurations locales liées à l'ordre directionnel. La cinétique de mise en équilibre thermique de ces configurations est relativement lente et ces configurations d'équilibre, stables à haute température, peuvent être figées par des trempes à température ambiante. Des mesures magnétiques relativement simples permettent de suivre globalement ces réarrangements cationiques locaux. Leur compréhension permet de contrôler les compositions et les traitements thermiques conduisant aux propriétés optimales souhaitées.

\section{REFERENCES}

[1] CHASSAING I., PRESMANES L., TAILHADES Ph., ROUSSET A., Sol. State Ionics (à paraître).

[2] BLASSE G., Philips Res. Reports suppl. 3 (1964) 72.

[3] NEEL L., J. Phys. Rad. 15 (1954) 225.

[4] TAILHADES Ph., MOLLARD P., ROUSSET A., GOUGEON M., IEEE Trans. Magn. MAG26 (1990) 1822.

[5] CHIKAZUMI S., Physics of Magnetism, chap.17, Ed. John Wiley Sons (1964).

[6] MOLLARD P., TAILHADES Ph, ROUSSET A., IEEE Trans. Mag. 26(1) (1990) 241.

[7] TAILHADES Ph., SARDA Ch., MOLLARD P., ROUSSET A., J. Magn. Magn. Mater. 104-107 (1992) 969.

[8] DUNITZ J.D., ORGEL L.E., J. Phys. Chem. Solids 3 (1957) 318.

[9] SAWATSKY G.A., van der WOUDE F., MORRISH A.H., J. Appl. Phys. 39 (1968) 1204.

[10] SAWATSKY G.A., van der WOUDE F., MORRISH A.H., Phys. Rev. 187 (1969) 747.

[11] DUPRE G.,Thèse Lyon (1976).

Remerciements: Ce travail s'intégre dans le cadre du programme scientifique du laboratoire mixte ODIL associant l'Université P. Sabatier, la société ATG Gigadisc et la Région Midi Pyrénées. Les auteurs remercient le Conseil Régional de Midi Pyrénées pour son soutien financier. 